\title{
Síntesis de estirilquinolinas con potencial Leishmanicida in vitro sobre Leishmania (Viannia) panamensis
}

\author{
Omar L. Torres ${ }^{(1) \star}$, Fernis J. Marin(1), Gilmar G. Santafé(2) y Sara M. Robledo(3) \\ (1) Fac. de Ciencias de la Salud, Dpto. de Regencia y Farmacia, Universidad de Córdoba, Cra 6 No. 76-103, Montería- \\ Colombia. (e-mail: omart2365@gmail.com; marinfj30@gmail.com) \\ (2) Fac. de Ciencias Básicas, Dpto. de Química, Universidad de Córdoba, Cra 6 No. 76-103, Montería-Colombia. \\ (e-mail: gsantafe@correo.unicordoba.edu.co) \\ (3) Fac. de Medicina, PECET-Instituto de Investigaciones Médicas, Universidad de Antioquia, Calle 62 No $52-59$ SIU \\ Laboratorio 632, Medellín-Colombia. (e-mail: sara.robledo@udea.edu.co)
}

* Autor a quien debe ser dirigida la correspondencia.

Recibido Jun. 7, 2019; Aceptado Ago. 5, 2019; Versión final Oct. 1, 2019, Publicado Feb. 2020

\begin{abstract}
Resumen
A partir de la reacción de condensación tipo Perkin entre quinaldina y la 8-hidroxiquinaldina con aldehídos aromáticos se sintetizaron tres estirilquinolinas: (E)-2-etoxi-4-(2-(8-hidroxiquinolin-2-il)vinil)fenil acetato (1), 2-[(E)-2-(2-acetiloxi-5-nitrofenil)etenil] quinolina (2), 2-[(E)-2-(3-metoxifenil)etenil] quinolina (3). Se determinó potencial leishmanicida in vitro empleando citometría de flujo y citotoxicidad por el método MTT. Los compuestos (1) y (2) mostraron ser activos contra Leishmania (V) panamensis con valores de concentración letal $50 \%\left(\mathrm{CL}_{50}\right)$ de 0.2 y $2.5 \mu \mathrm{g} / \mathrm{mL}$, $\mathrm{CL}_{50}$ de 2.8 y $5.9 \mu \mathrm{g} / \mathrm{mL}$ e índices de selectividad de 14.0 y 2.4 , respectivamente, comparados con el medicamento control anfotericina B. Las estructuras de estos compuestos se confirmaron empleando técnicas espectroscópicas de análisis como $I R, R M N-{ }^{1} \mathrm{H}$ y ${ }^{13} \mathrm{C}$, COSY, HMQC, HMBC y experimentos DEPT. Se sintetizaron tres estirilquinolinas con rendimientos de $40.3 \%, 72.0 \%$ y $80.0 \%$ respectivamente, las estirilquinolinas (1) y (2) mostraron buen potencial leishmanicida con porcentajes de inhibición de 67.5 y 74.9 respectivamente.
\end{abstract}

Palabras clave: estirilquinolinas; Leishmania; reacción tipo Perkin; potencial leismanicida

\section{Sinthesis of styrylquinolines with Leishmanicidal potential in vitro on Leishmania (Viannia) panamensis}

\begin{abstract}
A Perkin type condensation reaction between quinaldine and 8-hydroxyquinaldine with aromatic aldehydes, three styrylquinolines were synthesized: (E)-2-ethoxy-4-(2-(8-hydroxyquinolin-2-il)vinyl)phenyl acetate (1), 2[(E)-2-(2-acetiloxy-5-nitrophenyl)ethenyl] quinoline (2), 2-[(E)-2-(3-methoxyiphenyl)ethenyl] quinoline (3). The leishmanicidal potential in vitro employing flow cytometry was determined and the cytotoxicity by MTT method, was evaluated. The compounds (1) y (2) showed to be active against Leishmania (V) panamensis with lethal dose 50\%) (LD50) de 0.2 and $2.5 \mu \mathrm{g} / \mathrm{mL}$, LD $\mathrm{L}_{50}$ de 2.8 and $5.9 \mu \mathrm{g} / \mathrm{mL}$ and selectivity index of 14.0 and 2.4, respectively, compared to the drug control amphotericin $\mathrm{B}$. The structures of these compounds were confirmed using spectroscopic techniques from analysis: IR, ${ }^{1} \mathrm{H}$ and ${ }^{13} \mathrm{C}$ NMR, COSY, HMQC, HMBC and DEPT experiments. Three styrylquinolines were synthesized with performance of $40.3 \%, 72.0 \%$ and $80.0 \%$ respectively, styrylquinolines (1) and (2) showed good leishmanicidal potential with inhibition percentages of 67.5 y 74.9 respectively.
\end{abstract}

Keywords: styrylquinolines; Leishmania; Perkin reaction; leishmanicidal potential 


\section{INTRODUCCIÓN}

En el continente americano durante el año 2016 los casos de leishmaniasis se incrementaron en un 5\% en relación a años anteriores, debido principalmente a la incidencia e incremento de la enfermedad en países como Colombia, Perú y Nicaragua (WHO, 2018). Específicamente en Colombia, durante el año 2018 se registraron 5.577 casos de leishmaniasis (Instituto Nacional de Salud, 2018), y en lo que va corrido del año 2019 hasta la quinta semana epidemiológica se reportan más de 307 casos (Instituto Nacional de Salud, 2019), esta enfermedad es transmitida por la picadura de insectos del género Lutzomyia y se estima que el $91 \%$ de los municipios en Colombia que se encuentran por debajo de 1750 m.s.n.m han presentado casos de leishmaniasis, notándose un incremento gradual en la transmisión de la enfermedad en las ciudades debido al desplazamiento de los vectores y de las poblaciones a áreas semirurales (Marín et al., 2016, 2018). Dentro de las tres formas de la leishmaniasis, la leishmaniasis Cutánea Americana (ACL) es la forma más común y contagiosa no infecciosa, causada por parásitos del género Leishmania, esta se compone de un amplio espectro de enfermedades que van desde lesiones externas localizadas hasta leishmaniasis mucocutánea que son deformantes y que pueden ser fatales (Pinheiro et al., 2016).

Distintas sustancias han sido implementadas y evaluadas como medicamentos contra la leishmaniasis; las principales están dirigidas a inhibir el proceso de las rutas metabólicas específicas del parásito (Figueredo et al., 2014), sin embargo los medicamentos propuestos para el tratamiento de leishmaniasis han sido compuestos antimoniales pentavalentes (Sb5+), cuyo mecanismo de acción frente a Leishmania es controvertido y poco entendido, mientras que la anfotericina B, pentamidina, paromomicina y miltefosina son sustancias que se emplean actualmente en los tratamientos terapéuticos, pero presentan varios problemas principalmente relacionados con sus efectos secundarios, altos precios del producto y la complicada producción o síntesis específica del medicamento (Calixto et al., 2016). Toda esta problemática sumada a los fenómenos de resistencia del parásito al medicamento y a la carencia de una vacuna contra esta enfermedad hace necesaria la búsqueda de nuevas alternativas terapéuticas para solucionar este problema de salud pública (WHO, 2010, 2018).

Debido a la limitada disponibilidad de productos farmacéuticos efectivos para el tratamiento de la leishmaniasis, los estudios actuales están dirigidos a la elaboración de nuevas moléculas activas contra la enfermedad, mediante procesos de síntesis química tomando como base compuestos heterocíclicos como la quinaldina y la 8-hidroxiquinaldina para la obtención de compuestos con núcleos quinolínicos, los cuales se caracterizan por tener una amplia actividad antiprotozoaria, razón por la cual el interés de estos compuestos ha crecido exponencialmente en las últimas dos décadas, estableciéndose como estructuras privilegiadas para ser designadas como candidatas de nuevos medicamentos. Algunos estudios demuestran también que estos compuestos quinolínicos presentaron actividades anticancerígenas (Mrozek et al., 2015), antibacterial, antifúngica y de neuroprotección con resultados promisorios debido a la gran versatilidad de la 8-hidroxiquinolina para formar una gran cantidad de compuestos derivados mediante uniones con metales, así como con sistemas nanoparticulados (Oliveri, 2016). Por su parte, Soto et al. (2011), realizaron la síntesis de seis análogos de estirilquinolinas con ausencia de efecto citotóxico frente a la línea celular Jurkat de origen leucemoide, estableciendo en su investigación una opción para tratamientos biomédicos como antiproliferativos y/o anticancerígenos y constituirse en una alternativa para el tratamiento de la leishmaniasis basados en la fabricación y evaluación de moléculas de origen sintético.

Como respuesta a la obtención de sustancias heterocíclicas quinolínicas o quinaldínicas se ha venido implementando la reacción de condensación tipo Perkin a partir de quinaldina e hidroxiquinaldina con algunos aldehídos aromáticos, mediante la cual Torres et al. (2014), sintetizaron 5 estirilquinolinas (Q), dentro de las cuales los compuestos Q.1 y Q.5 mostraron una buena actividad antimalárica in vitro con IC50 de 6.4 y $10.8 \mu \mathrm{M}$ y con una moderada y baja citotoxicidad, respectivamente, comparado con el medicamento control Amodiaquina cuyo $\mathrm{IC}_{50}$ es $0.0345 \mu \mathrm{M}$. Sánchez et al. (2014), sintetizaron y caracterizaron tres estirilquinolinas, las cuales mostraron mediante evaluación in vitro que el compuesto 2[(E)-2-(2,3-diacetil-oxifenil)etenil] quinolina presentó una gran actividad contra Leishmania (Viannia) panamensis con un $\mathrm{IC}_{50}$ de $1.4 \mu \mathrm{g} / \mathrm{mL}$ e índice de selectividad (IS) de 3.9. Posteriormente mediante la aplicación del mismo método se sintetizó la estirilquinolína 2-[(E)-2-(4-isopropilfenil) etenil] quinolina que presentó gran actividad antimalárica in vitro con valores $\mathrm{IC}_{50}$ de $38.46 \mu \mathrm{g} / \mathrm{mL}$ frente a FCB-2 resistente a la cloroquina y $33.70 \mu \mathrm{M}$ frente a la cepa NF-54 sensible a la cloroquina (Santafé et al, 2016). De acuerdo con lo expuesto anteriormente, es importante continuar sintetizando moléculas tipo estirilquinolinas con el fin de encontrar compuestos con mejor actividad sobre amastigotes intracelulares de L. (V) panamensis. En esta investigación se realizó la síntesis de tres estirilquinolinas, a partir de quinaldina y 8 - hidroxiquinaldina con diferentes aldehídos aromáticos evaluando el potencial leishmanicida y la citotóxidad de estas moléculas en células U937. 


\section{METODOLOGÍA}

La metodología se presenta en dos secciones: Sección 1, en la que se describe el procedimiento químico de la síntesis de las estirilquinolinas y Sección 2, en la que se describe el ensayo de citotoxicidad sobre macrófagos de la línea celular U937 y la evaluación de la actividad leishmanicida de los compuestos sintetizados.

\section{Sección 1. Síntesis de estirilquinolinas}

La síntesis de las estirilquinolinas (E)-2-etoxi-4-(2-(8-hidroxiquinolin-2-il)etenil)fenil acetato (1), 2-[(E)-2-(2acetiloxi-5-nitrofenil)etenil] quinolina (2), 2-[(E)-2-(3-metoxifenil)etenil] quinolina (3), se realizó mediante la reacción de condensación tipo Perkin (Fig. 1.) a partir de quinaldina, anhídrido acético y los aldehídos 3etoxi-4-hidroxibenzaldehido, 2-hidroxi-5-nitrobenzaldehido y 3-metoxibenzaldehido, respectivamente. Todos los reactivos usados fueron grado analítico (Merck).<smiles>[R]c1ccc2nc(C)ccc2c1</smiles>

Quinaldina o 8-hidroxiquinaldina

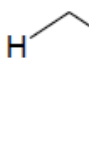<smiles>[R][Te]1(C)C=CC=CC=C1</smiles>

Aldehído aromático

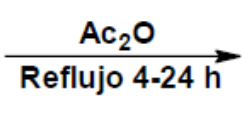

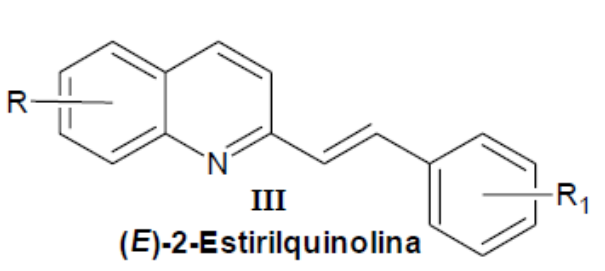

Fig. 1: Reacción de Condensación tipo Perkin.

Preparación de las soluciones de síntesis

Para la obtención de estirilquinolinas, se preparó una solución añadiendo $7.0 \mathrm{mmol}$ de quinaldina en $12 \mathrm{~mL}$ de anhídrido acético y posteriormente se adicionó el aldehído aromático correspondiente. La mezcla de reacción se sometió a reflujo por intervalos de tiempos que oscilaron entre 6 y 24 horas y se monitoreo periódicamente empleando cromatografía en capa delgada (CCD). Una vez finalizada la reacción se dejó enfriar hasta temperatura ambiente con posterior neutralización por adición de solución saturada de bicarbonato de sodio $\mathrm{NaHCO}_{3}$, hasta obtener la hidrólisis completa; luego se adicionaron $50 \mathrm{~mL}$ de hexano: acetato de etilo en proporción 1:2 para extraer el derivado quinolínico por cromatografía de reparto con repeticiones sucesivas. La fase orgánica se secó con sulfato de sodio anhidro y posteriormente se concentró a presión reducida. Luego el producto crudo se purificó por cromatografía en columna (CC), utilizando como eluyente bencina de petróleo: acetato de etilo con gradiente de polaridad creciente. (Torres et al., 2014; Sánchez et al., 2014).

\section{Identificación de sustancias}

Las estructuras de los compuestos purificados fueron confirmadas utilizando las técnicas espectroscópicas IR (Perkin-Elmer RX/FT-IR System), RMN-1 H, RMN-13 C (Brucker $400 \mathrm{MHz}$; $\mathrm{CDCl}_{3}$ ), experimentos bidimensionales (DEPT, COSY, HMQC, HMBC) y por comparación con datos de estructuras similares reportados por otros autores (Soto et al., 2011; Torres et al., 2014).

\section{Sección 2. Ensayo de citotoxicidad en la línea celular U937 y evaluación de la actividad leishmanicida}

Para la evaluación de la actividad citotóxica se realizaron 6 diluciones dobles seriadas preparadas a partir de una solución madre de $200 \mu \mathrm{g} / \mathrm{mL}$ en dimetilsulfóxido (DMSO) (Sigma-Aldrich, St Louis MO, USA), equivalentes a $100,50,10,2.5,0.625$ y $0.156 \mu \mathrm{g} / \mathrm{mL}$ para las estirilquinolinas sintetizadas.

Para la evaluación de la actividad leishmanicida inicialmente se preparó una solución a $20 \mu \mathrm{g} / \mathrm{mL}$., luego, para los compuestos con alguna actividad a esta concentración se prepararon diluciones cuádruples seriadas desde 100.0 hasta $1.562 \mu \mathrm{g} / \mathrm{mL}$. Para la anfotericina B se prepararon cuatro soluciones a partir de $1.0 \mu \mathrm{g} / \mathrm{mL}$. Todas las soluciones se hicieron en DMSO al $0.5 \%$ y medio RPMI 1640 (Gibco, Thermo Scientific Inc., Waltham, MA, USA), suplementado con $10 \%$ de suero fetal bovino (SFB) (Gibco) y $1 \%$ de antibióticos (penicilina-estreptomicina) (Sigma).

\section{El parásito}

En los ensayos de actividad leishmanicida in vitro se utilizó la cepa de de $L$. (V) panamensis (UA140-pIR(-)eGFP) cultivados como promastigotes en medio bifásico NNN modificado (Novy-MacNeil-Nicolle), empleando como fase sólida agar sangre (Merck Millipore Corporation, Medellín, Colombia) y como fase 
líquida solución salina de buffer fosfato (PBS) más glucosa, pH 6.9 e incubados a $26^{\circ} \mathrm{C}$. Los promastigotes se usaron posteriormente para infectar los macrófagos U937 y obtener el estadio de amastigotes intracelulares. Con el fin de asegurar una mayor infección de los macrófagos in vitro, la cepa de $L$. (V) panamensis se mantuvo en hámster (Mesocricetus auratus) infectados experimentalmente. Previamente a la infección in vitro, se hizo un aspirado de la lesión del hámster con solución de fosfatos (PBS) y aguja \#26; luego, la muestra aspirada se cultivó en medio NNN e incubados a una temperatura de $27^{\circ} \mathrm{C}$ hasta obtener promastigotes.

\section{Línea celular}

Monocitos humanos U937 (CRL-1593-2 ${ }^{\mathrm{TM}}$ ), adquirida del ATCC (American Type Cell Collection) se mantuvieron en medio RPMI- 1640 completo a $37^{\circ} \mathrm{C}$, con $5 \%$ de $\mathrm{CO}_{2}$.

\section{Actividad citotóxica sobre células U937}

La citotoxicidad se evaluó sobre las células U-937 empleando el micrométodo enzimático con bromuro de 3(4,5-dimetiltiazol-2-il)-2,5-difeniltetrazolio (MTT). Las células en fase exponencial de crecimiento se ajustaron a una concentración de 100.000 células/mL de medio RPMI-1640 suplementado con SFB y antibióticos. En cada pozo de una placa para cultivo celular de 96 pozos (Falcon, Fisher Scientific, Thermo Scientific Inc, Waltham, MA, USA) se depositaron $100 \mu \mathrm{l}$ de células. Luego se adiciono en cada pozo $100 \mu \mathrm{l}$ de cada una de las concentraciones del respectivo compuesto. Como control negativo (viabilidad o no citotoxicidad) se usaron células en medio RPMI completo y mantenidas bajo las mismas condiciones de incubación, pero en ausencia de cualquier compuesto; como control de citotoxicidad se utilizaron células expuestas a anfotericina B.

Las células en presencia de las diferentes soluciones de los compuestos, así como los controles se incubaron a $37^{\circ} \mathrm{C}$ en una atmósfera al $5 \%$ de $\mathrm{CO}_{2}$ durante 72 horas. Transcurrido el período de incubación, el efecto de los compuestos sobre las células se determinó midiendo la actividad de la enzima mitocondrial succinato deshidrogenasa. Para ello, se adicionó, $10 \mu$ l/pozo de una solución de MTT con concentración 5 $\mu \mathrm{g} / \mathrm{mL}$ (Sigma) y se incubaron a $37^{\circ} \mathrm{C}$ durante 3 horas. Pasado este período de incubación se adicionaron $100 \mu \mathrm{l} /$ pozo de una solución de $50 \%$ de isopropanol (Merck Millipore) y $10 \%$ de dodecil sulfato de sodio (SDS) (Merck Millipore) para solubilizar los cristales de formazán (Insuasty et al., 2015). Los platos se incubaron durante otros 30 minutos y la producción de formazán (que es proporcional al porcentaje de células viables) se midió en un lector de microplacas (Benchmark Bio-Rad Hercules CA, USA) a una densidad óptica de $570 \mathrm{~nm}$.

La citotoxicidad se determinó de acuerdo al porcentaje de disminución de la viabilidad y por ende, de la disminución en la cantidad de células obtenidas para cada extracto o compuesto y la anfotericina $\mathrm{B}$ según las densidades ópticas (D.O) obtenidas en cada condición experimental. La disminución de la viabilidad celular denominada inhibición del crecimiento celular, se calculó utilizando los valores de D.O para cada condición: compuesto o control a la concentración evaluada, usando la siguiente ecuación: \% Viabilidad = [D.O células expuestas al compuesto o control $\div$ D.O células no expuestas] $\times 100$ ). Los valores de D.O obtenidas para las células en ausencia de compuestos corresponde al $100 \%$ de viabilidad o células vivas. Luego, con los porcentajes de viabilidad se calculó el porcentaje de mortalidad, que corresponde a 100 - \% viabilidad. Por último, con los porcentajes de mortalidad se calculó la concentración letal $50\left(\mathrm{CL}_{50}\right)$ por el método de análisis dosis-respuesta, Probit (Finney, 1971) usando el programa estadístico SAS Data Analysis (SAS Institute Cary NC, USA). Los ensayos se realizaron dos veces con tres réplicas para cada concentración evaluada. La citotoxicidad de cada compuesto se clasificó según los valores de CL50 usando una escala propia: citotoxicidad alta $\mathrm{CL}_{50}<50 \mu \mathrm{g} / \mathrm{mL}$; citotoxicidad moderada: $\mathrm{CL}_{50}>50$ a $<200 \mu \mathrm{g} / \mathrm{mL}$ y citotoxicidad baja: $\mathrm{CL}_{50}>200 \mu \mathrm{g} / \mathrm{mL}$.

\section{Actividad sobre L. (V) panamensis}

La actividad de los compuestos se evaluó sobre amastigotes intracelulares obtenidos luego de la infección in vitro de macrófagos U937 diferenciados con miristato acetato de forbol (PMA del inglés phorbol myristate acetate) (Sigma). Para ello, las células U937 mantenidas en cultivo en suspensión se centrifugaron a 1,500 rpm durante 10 minutos y luego de descartar el sobrenadante, el botón de células se resuspendió a una concentración de $1 \times 10^{5}$ células $/ \mathrm{mL}$ de medio RPMI 1640 al $10 \%$ SFB con $0.1 \mu \mathrm{g} / \mathrm{mL}$ de PMA. En cada pozo de una placa para cultivo celular de 24 pozos se dispersó $1 \mathrm{~mL}$ de la suspensión de células y se incubaron a una temperatura de $37^{\circ} \mathrm{C}$ con una atmosfera al $5 \% \mathrm{CO}_{2}$. Transcurridas 48 horas de incubación las células se infectaron con promastigotes en fase estacionaria de crecimiento en una proporción 15:1 parásito: célula. Los platos se incubaron a $34^{\circ} \mathrm{C}$ en una atmosfera al $5 \%$ de $\mathrm{CO}_{2}$ durante 2 horas. Posteriormente se realizaron 2 lavados con PBS para eliminar los parásitos libres, se adicionó $1 \mathrm{~mL}$ de medio RPMI 1640 
completo y las células se incubaron nuevamente durante 24 horas. Pasado este tiempo, las células infectadas se expusieron a cada una de las concentraciones de los compuestos durante 72 horas. Como control de infección se usaron células infectadas y cultivadas en ausencia de los compuestos y como control de efectividad se empleó la anfotericina B. Luego de 72 horas de incubación a $34^{\circ} \mathrm{C}$ en una atmósfera al $5 \%$ de $\mathrm{CO}_{2}$ las células se removieron cuidadosamente del fondo del plato utilizando el émbolo de una jeringa y se analizaron en un citómetro de flujo leyendo a $488 \mathrm{~nm}$ de excitación y $525 \mathrm{~nm}$ de emisión con un láser de Argón (Galeano et al., 2011). Cada concentración de los compuestos y de los controles se evaluó por triplicado en dos experimentos diferentes. La actividad anti-Leishmania se determinó de acuerdo a la carga parasitaria, que es la cantidad de parásitos en las células infectadas obtenidas para cada concentración de cada extracto, compuesto y anfotericina B según el número de eventos positivos para la fluorescencia verde usando diagrama de puntos e histogramas según la intensidad media de fluorescencia (IFM) (Pulido et al., 2012).

La disminución de la carga parasitaria, denominada inhibición de la infección, se calculó utilizando los valores de IFM para cada condición, es decir, compuesto o control a la concentración evaluada, usando la siguiente ecuación: \% Infección = [IFMI células infectadas y expuestas al compuesto o control $\div$ IFM células infectadas y no expuestas] $\times$ 100). Los valores de IFM obtenidas para las células infectadas en ausencia de compuestos corresponde al $100 \%$ de parásitos. A su vez, el porcentaje de inhibición de la infección corresponde a 100 - \% infección

Para los compuestos que mostraron alguna inhibición a $20 \mu \mathrm{g} / \mathrm{mL}$, se determinó también la Concentración Efectiva 50 (EC50) calculada por el método Probit (Finney, 1971). Al igual que la citotoxicidad, la actividad anti-Leishmania se clasificó según los valores de $C_{50}$ así: actividad alta: $C_{50}<25 \mu \mathrm{g} / \mathrm{mL}$; actividad moderada: $\mathrm{CE}_{50}>25 \mathrm{a}<50 \mu \mathrm{g} / \mathrm{mL}$ y actividad baja: $\mathrm{CE}_{50}>50 \mu \mathrm{g} / \mathrm{mL}$. Finalmente, la actividad citotóxica se correlacionó con la actividad anti-Leishmania calculando el Índice de Selectividad (IS) con la siguiente ecuación: $\mathrm{IS}=\mathrm{CL}_{50} \div \mathrm{CE}_{50}$.

\section{RESULTADOS Y DISCUSIÓN}

Se sintetizaron tres estirilquinolinas: (E)-2-etoxi-4-(2-(8-hidroxiquinolin-2-il)vinil)fenil acetato (1), (E)-4-nitro-2(2-quinolin-2-il)vinil)fenil acetato (2), (E)-2-(3-metoxiestiril)]quinolina (3), como se muestra en la Figura 2.<smiles>CCOc1cc(/C=C/c2ccc3cccc(O)c3n2)ccc1OC(C)=O</smiles>

(E)-2-etoxi-4-(2-(8-hidroxiquinolin-2-il)vinil)fenil acetato<smiles>CC(=O)Oc1ccc([N+](=O)[O-])cc1/C=C/c1ccc2ccccc2n1</smiles>

(E)-4-nitro-2-(2-(quinolin-2-il)vinil)fenil acetato

(1)

(2)<smiles>COc1cccc(/C=C/c2ccc3ccccc3n2)c1</smiles>

(E)-2-(3-metoxiestiril)-quinolina

(3)

Fig. 2: Estructuras químicas de las estirilquinolinas sintetizadas

(E)-2-etoxi-4-(2-(8-hidroxiquinolin-2-il)vinil)fenil acetato (1): Sólido amarillo; $\mathrm{C}_{20} \mathrm{H}_{17} \mathrm{O}_{3} \mathrm{~N}$; Rendimiento $45 \%$. RMN-1 ${ }^{1} \mathrm{H}\left(300 \mathrm{MHz}, \mathrm{CDCl}_{3}\right) ; \delta_{\mathrm{H}}: 8.15(\mathrm{~d}, \mathrm{~J}=8.5 \mathrm{~Hz}, 1 \mathrm{H}, \mathrm{H} 4) ; 7.66$ (d, J=8.5 Hz, 1H, H3); $\delta_{\mathrm{H}:} 7.70$ (d, J=16.0

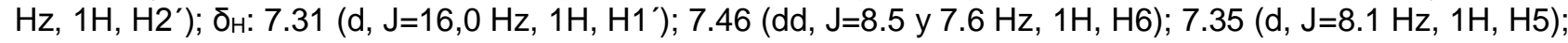
$7.23(\mathrm{~m}, 1 \mathrm{H}, \mathrm{H} 7)$; señales de protones del anillo aromático en $\delta_{\mathrm{H}:} 7.14\left(\mathrm{~d}, \mathrm{~J}=7.9 \mathrm{~Hz}, \mathrm{H} 5\right.$ ”); $\delta_{\mathrm{H}}:$ 7.19-7.27 (m, $2 \mathrm{H}, \mathrm{H} 2$ " y H6"); $\delta_{\mathrm{H}}: 2.41$ (s, $3 \mathrm{H},-\mathrm{CH}_{3}$ ) protones de un grupo acetiloxi y $\delta_{\mathrm{H}}: 4.20\left(\mathrm{q}, \mathrm{J}=6.9 \mathrm{~Hz}, 2 \mathrm{H},-\mathrm{CH}_{2}-\right)$ y $\delta_{\mathrm{H}}$ : $1.51\left(\mathrm{t}, \mathrm{J}=6.9 \mathrm{~Hz}, 3 \mathrm{H},-\mathrm{CH}_{3}\right)$, protones del grupo etoxilo. $\mathrm{RMN}-{ }^{13} \mathrm{C}\left(75 \mathrm{MHz}, \mathrm{CDCl}_{3}\right)$ y DEPT-135: $\delta_{\mathrm{c}}: 169.1$

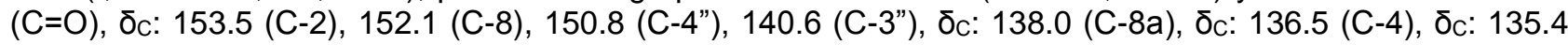




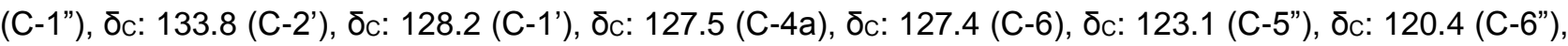

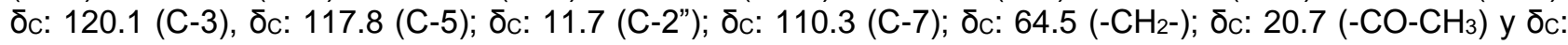
$14.8\left(\mathrm{OR}-\mathrm{CH}_{3}\right) \mathrm{ppm}$.

(E)-4-nitro-2-(2-quinolin-2-il)vinil)fenil acetato (2): Sólido amarillo; $\mathrm{C}_{19} \mathrm{H}_{14} \mathrm{O}_{4} \mathrm{~N}_{2}$; Rendimiento $72 \%$. RMN-1 $\mathrm{H}$ $\left(300 \mathrm{MHz}, \mathrm{CDCl}_{3}\right) \delta_{\mathrm{H}}: 8.16\left(\mathrm{~s}, 1 \mathrm{H}, \mathrm{H6} 6^{\prime}\right) ; \delta_{\mathrm{H}}: 8.25$ (d, J=7.6 y $\left.2.3 \mathrm{~Hz}, 1 \mathrm{H}, \mathrm{H} 4^{\prime}\right) ; \delta_{\mathrm{H}}: 8.25$ (d, J=8.5 Hz, $\left.1 \mathrm{H}, \mathrm{H} 4\right)$; $\delta_{H: ~} 8.19$ (d, J=8.4 Hz, 1H, H8); $\delta_{H}: 7.89$ (d, J=16.1 Hz, 1H, H2"); $\delta_{H}: 7.88$ (m, 1H, H5); $\delta_{H}: 7.81$ (d, J=8.4 Hz, $1 \mathrm{H}, \mathrm{H7}$ ); $\delta_{H}: 7.69$ (d, J=8.5 Hz, 1H, H3); $\delta_{H}: 7.61$ (t, J=7.3 Hz, 1H, H6); $\delta_{H}: 7.59$ (d, J=16.1 Hz, 1H, H1"); $\delta_{H}$ : $7.41\left(\mathrm{~d}, \mathrm{~J}=8.9 \mathrm{~Hz}, 1 \mathrm{H}, \mathrm{H} 3^{\prime}\right) ; \delta_{\mathrm{H}}: 2.56\left(\mathrm{~s}, 1 \mathrm{H}, \mathrm{CH}_{3}\right)$ de un grupo acetiloxi. RMN- ${ }^{13} \mathrm{C}\left(75 \mathrm{MHz}, \mathrm{CDCl}_{3}\right)$ y DEPT135: $\delta_{c}: 168.4(C=O), \delta_{c}: 154.4(C-2), \delta_{c}: 152.8(C-2)$, $\delta_{c}: 148.2$ (C-8a), $\delta_{c}: 145.8$ (C-5'), $\delta_{c}: 137.0(C-4), \delta_{c}:$ 133.3 (C-1"), $\delta_{c}: 130.7$ (C-1'), $\delta_{c}: 130.2$ (C-7), $\delta_{c}: 129.2$ (C-8), $\delta_{c}: 127.7$ (C-4a), $\delta_{c}: 127.6$ (C-5), $\delta_{c}: 126.9$

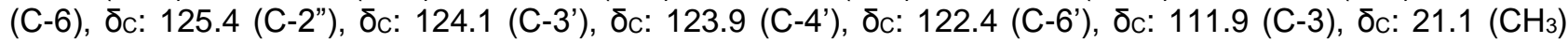
ppm.

(E)-2-(3-metoxiestiril)] quinolina (3): Cristales blancos; $\mathrm{C}_{18} \mathrm{H}_{15} \mathrm{ON}$; Rendimiento $80 \%$. $\mathrm{RMN}-{ }^{1} \mathrm{H}$ (300 MHz, $\mathrm{CDCl}_{3}$ ) $\delta_{\mathrm{H}}: 8.14$ (d, J=8.4 Hz, 1H, H4); $\delta_{H}: 7.70$ (d, J=8.4 Hz, 1H, H8); $\delta_{H}: 7.74$ (d, J=8.4 Hz, 1H, H7); $\delta_{H}$ : 7.67 (d, J=16.3 Hz, 1H, H2"); $\delta_{H}: 7.45$ (d, J=16.3 Hz, 1H, H1"); $\delta_{H}: 7.27$ (s, 1H, H2'); $\delta_{H}: 7.35$ (t, J= 8.0 y 7.8 $\left.\mathrm{Hz}, 1 \mathrm{H}, \mathrm{H} 5^{\prime}\right) ; \delta_{\mathrm{H}}: 7.23$ (d, J=7.8 Hz, 1H, H6'); $\delta_{\mathrm{H}}: 3.89$ (s, $\left.1 \mathrm{H}, \mathrm{OCH}_{3}\right) . \mathrm{RMN}^{13} \mathrm{C}\left(75 \mathrm{MHz}, \mathrm{CDCl}_{3}\right)$ y DEPT-

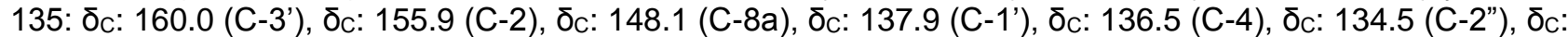

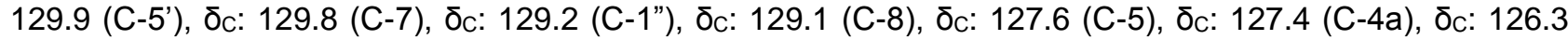

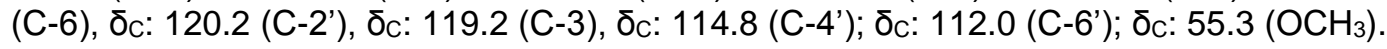

La asignación de las estructuras se realizó mediante el análisis de los datos espectroscópicos, apoyados en reportes similares de otros autores (Soto et al., 2011; Torres et al., 2014; Santafé et al., 2016).

\section{Evaluación del potencial leishmanicida y citotóxico}

Los resultados de la evaluación del potencial leishmanicida in vitro frente a amastigotes intracelulares de $L$. (V) panamensis UA140-pIR(-)-eGFP y de la citotoxicidad en la línea celular U937, de las diferentes estirilquinolinas sintetizadas se resumen en la Tabla 1.

Tabla 1: Potencial leishmanicida y actividad citotóxica de las estirilquinolinas sintetizadas 1 - 3 .

\begin{tabular}{lllll}
\hline \multirow{2}{*}{ Estirilquinolinas } & $\begin{array}{l}\mathrm{CL} 50(\mu \mathrm{g} / \mathrm{mL}) \\
\mathrm{X} \pm \mathrm{SD}\end{array}$ & $\begin{array}{l}\text { \% Inhibición } \\
\mathrm{X} \pm \mathrm{SD}\end{array}$ & $\begin{array}{l}\mathrm{CE}_{50}(\mu \mathrm{g} / \mathrm{mL}) \\
\mathrm{X} \pm \mathrm{SD}\end{array}$ & \multirow{2}{*}{$\mathrm{IS}$} \\
\hline 1 & $2.8 \pm 0.3$ & $67.5 \pm 6.5^{\star \star}$ & $0.2 \pm 0.0$ & 14.0 \\
2 & $5.9 \pm 0.5$ & $74.9 \pm 4.1^{\star \star}$ & $2.5 \pm 0.4$ & 2.4 \\
3 & $0.8 \pm 0.2$ & 5.5 & $\mathrm{NE}$ & $<1$ \\
Anfotericina B & $52.5 \pm 3.6$ & $62.7 \pm 1.4^{\star}$ & $0.05 \pm 0.001$ & 1050 \\
\hline
\end{tabular}

En las Tabla 1, los datos representan el promedio \pm de la desviación estándar de la Concentración Letal 50 $(\mathrm{CL} 50)$, la Concentración Efectiva $50\left(\mathrm{CE}_{50}\right)$ en $\mu \mathrm{g} / \mathrm{mL}$, y el Porcentaje de Inhibición a una concentración de $20 \mu \mathrm{g} / \mathrm{mL}$. NE: No evaluado. ${ }^{*}$ Concentración evaluada: $0.5 \mu \mathrm{g} / \mathrm{mL}$; ${ }^{* *}$ Concentración evaluada: $3.0 \mu \mathrm{g} / \mathrm{mL}$ y ${ }^{* * * * \star}$ Concentración evaluada: $0.4 \mu \mathrm{g} / \mathrm{mL}$ Las estirilquinolinas (1), (2) y (3) con una CL50 de 2.8, 5.9 y 0.8 $\mu \mathrm{g} / \mathrm{mL}$ respectivamente, mostraron una alta citotoxicidad según la escala establecida, con valores de $\mathrm{CL}_{50}<$ $50 \mu \mathrm{g} / \mathrm{mL}$, comparados con el medicamento control anfotericina B cuya CL50 es de $52.5 \mu \mathrm{g} / \mathrm{mL}$.

De la misma manera se observa que las estirilquinolinas (1) y (2) presentaron un buen potencial leishmanicida contra los amastigotes intracelulares de Leishmania (V) panamensis mostrando valores de $\mathrm{CE}_{50}$ de 0.2 y $2.5 \mu \mathrm{g} / \mathrm{mL}$, lo cual permite clasificarlos como compuestos con alta actividad, siendo estos compuestos capaces de inhibir la carga parasitaria en un $67.5 \%$ y $74.9 \%$, respectivamente, dejando en evidencia que los compuestos (1) y (2) poseen una mayor capacidad inhibitoria comparado con la anfotericina B que presentó un porcentaje de inhibición del 62.7\%. Estas dos moléculas mostraron ser muy activas contra Leishmania (V) panamensis, con valores de Índices de selectividad de 14.0 y 2.4 , respectivamente, por lo cual las moléculas sintéticas (1) y (2) se consideran promisorias para el desarrollo de nuevos medicamentos, teniendo en cuenta su $I S>1$, de esta manera se demostró que para las estirilquinolinas (1) y (2) su potencial leishmanicida supera su capacidad citotóxica. Estos resultados son variables teniendo en cuenta la incidencia de los grupos sustituyentes unidos a la estructura de la estirilquinolína sintetizada, como se evidencia al hacer la comparación con las estirilquinolinas E1, E2 y E3 sintetizadas por Sánchez et al. (2014) según la Figura 3. 
<smiles>CCCCc1ccc2ccccc2n1</smiles>

Fig. 3: Estirilquinolinas sintetizadas E1, E2 y E3.

Comparando la estructura del compuesto (1) y el compuesto E2, se puede ver que las diferencias entre ellas radican básicamente en la presencia de un grupo hidroxilo $(\mathrm{OH})$ en la posición 8 del anillo quinolínico del compuesto (1), que no está presente en E2; así como un grupo etoxilo en la posición 2" del anillo aromático del compuesto (1), y que en el compuesto E2 corresponde a un grupo metoxilo, estas diferencias estructurales son las responsables de la gran actividad ( $C_{50}$ de $0.2 \mu \mathrm{g} / \mathrm{mL}$ ) y la gran selectividad $(\mathrm{IS}=14)$ presentada por el compuesto (1) contra $L$. (V) panamensis, así como de la alta toxicidad $\left(\mathrm{CL}_{50}=2.8 \mu \mathrm{g} / \mathrm{mL}\right)$; mientras que el compuesto E2 no fue activo contra $L$. (V) panamensis $\left(\mathrm{CE}_{50}\right.$ de $68.1 \mu \mathrm{g} / \mathrm{mL}$ e IS $<1$ ), pero resulto ser menos citotóxico que el compuesto (1), con una $\mathrm{CL}_{50}>68.1 \mu \mathrm{g} / \mathrm{mL}$, lo cual lo clasificó como moderadamente tóxico según la escala establecida.

El compuesto (2) presenta en su estructura un grupo nitro en posición para respecto al grupo acetilo del anillo aromático, mientras que en los compuestos E1 y E3 presentan grupos acetilos en posición orto con respecto a grupo acetilo principal del anillo aromático, estas diferencias en los grupos sustituyentes y posiciones en las estirilquinolinas muestran que en este caso el grupo acetilo en posición orto genera una mayor actividad leishmanicida para el compuesto E3 con una $\mathrm{CE}_{50}$ de $1.4 \mu \mathrm{g} / \mathrm{mL}$ y un IS de 3.9 , mientras que en el compuesto (2), el grupo nitro $\left(-\mathrm{NO}_{2}\right)$ sobre el anillo aromático produce una disminución en la acción contra $L$. (V) panamensis con una $\mathrm{CE}_{50}$ de $2.5 \mu \mathrm{g} / \mathrm{mL}$ y un IS de 2.4, reduciendo la capacidad para inhibir la carga parasitaria; el compuesto $\mathrm{E} 1$ no fue activo ni selectivo contra el parásito con una $\mathrm{CE}_{50}>5.6$ $\mu \mathrm{g} / \mathrm{mL}$ y un IS de $<1.0$. Además, los compuestos (2) y E1 presentaron alta citotoxicidad con CL50 entre 5.9 y $5.4 \mu \mathrm{g} / \mathrm{mL}$.

Coimbra et al. (2016), sintetizaron nuevos derivados quinolínicos dentro de los cuales el compuesto denominado QuinDer1 (Figura 4), presentó actividad en promastigotes de L. amazonensis y L. braziliensis, exhibiendo una fuerte inhibición de la proliferación de amastigotes de L. amazonensis a una $\mathrm{IC}_{50}$ de 0.022 $\mu \mathrm{g} / \mathrm{mL}$, siendo 139 veces más activo que miltefosina con $\mathrm{IC}_{50}$ de $5.176 \mu \mathrm{g} / \mathrm{mL}$, utilizado como fármaco de referencia. Este compuesto presentó baja citotoxicidad hacia los macrófagos murinos y los eritrocitos humanos. Además, los promastigotes de L. amazonensis tratados con el compuesto QuinDer1 mostraron una alta generación de niveles de ROS con bajas alteraciones en el potencial de la membrana mitocondrial y el mantenimiento de la integridad de la membrana del parásito.<smiles>NCCSc1ccnc2cc(Cl)ccc12</smiles>

Fig. 4: Derivado de quinolina QuinDer1.

Estos resultados evidencian la importancia de la unión y la posición de los grupos sustituyentes en sistema quinolínico, de tal forma que se puede ver la gran capacidad del compuesto QuinDer1 para inhibir el efecto de $L$. amazonensis a muy bajas concentraciones, efecto debido al grupo aminotiol en la posición para respecto al nitrógeno heteroátomo, así como a la presencia de un grupo Cloro en la posición 7 del anillo, estos sustituyentes son muy diferentes comparados con los sustituyentes de los compuestos 1,2 y 3 sintetizados en este trabajo, ya que en este estudio los sustituyentes unidos a la quinolina son $\mathrm{OH}$ en la posición 7 y sistemas aromáticos con grupos $-\mathrm{NO}_{2},-\mathrm{OCH}_{2} \mathrm{CH}_{3},-\mathrm{OCH}_{3},-\mathrm{OCOCH}_{3}$, además existe la presencia de un doble enlace. Todas las variaciones estructurales son factores que influyen en la manera en como los compuestos actúan sobre las diferentes especies del género Leishmania, caso que ocurre en esta comparación de trabajos de investigación ya que Coimbra et al. (2016), evaluaron sus moléculas sintéticas sobre promastigotes de $L$. amazonensis y L. braziliensis, mientras que los compuestos 1,2 y 3 se evaluaron sobre amastigotes intracelulares de Leishmania (V) panamensis, lo que indica que la acción de 
los compuestos difiere de una a otra especie, resaltando incluso la diferencia en los estadios del parásito durante el proceso de evaluación. Por otro lado Ribeiro et al. (2018), reportan la síntesis del compuesto AMQ-j derivado de la 4-hydrazinoquinoline quien exhibió un fuerte efecto contra $L$. amazonensis, quien fue efectivo en experimentos in vivo frente a en ratones BALB/c infectados experimentalmente; así mismo Abdelwahid et al. (2019), sintetizaron quince ácidos quinolin-4-carboxílicos (Q1-Q15), dentro de los resultados se resalta el compuesto Q1 (Ácido 2-metilquinolina-4-carboxílico) quien resultó ser muy activo contra $L$. donovani con un $\mathrm{IC}_{50}$ de $1.49 \mu \mathrm{g} / \mathrm{mL}$, el cual fue cinco veces más potente que el estibogluconato de sodio $\left(\mathrm{IC}_{50}=8.06 \mu \mathrm{g} / \mathrm{mL}\right)$ y diez veces más potente que la anfotericina $B\left(I C_{50}=14.70 \mu \mathrm{g} / \mathrm{mL}\right)$, usados como medicamentos de control (Figura 5), estos estudios soportan la importancia que representa el núcleo quinolina y los diferentes sustituyentes que se puedan adicionar, estas modificaciones estructurales permiten seguir en la búsqueda de una molécula que sea efectiva sobre los parásitos del género Leishmania .<smiles>Clc1ccc2c(NNc3ccccc3)ccnc2c1</smiles>

AMQ-j<smiles>Cc1cc(C(=O)O)c2ccccc2n1</smiles>

Q1

Fig. 5: Compuestos AMQ-j y Q1.

Sin embargo, estos estudios son bases fundamentales para continuar en la búsqueda de moléculas que permitan mitigar los daños causados a la salud por la leishmaniasis como una de las enfermedades huérfanas que lesionan las comunidades más pobres del planeta.

De igual forma Mesa et al. (2008) reporta la síntesis de tres estirilquinolinas: 2-[(E)-2-(2,5-dimetoxi-fenil) etenil] quinolina, 2-[(E)-2-(2,3-dimetoxifenil)etenil] quinolina y $\mathrm{N}-\{4-[(E)-2$-quinolin2-iletenil]fenil\} acetamida, con valores de $\mathrm{CE}_{50}$ de $1.4,1.8$ y $1.7 \mu \mathrm{g} / \mathrm{mL}$ e IS de $1.0,1.2$ y 0.2 , respectivamente, siendo cada uno de estos compuestos altamente activos contra amastigotes axénicos de $L$. panamensis y que son compuestos con variación en los grupos sustituyentes, sin embargo comparándolos con el compuesto (1) se puede notar que este presenta una mayor actividad y selectividad ( $C E_{50}$ de 0.2 e IS de 14.0), evidenciando de esta manera que los sustituyentes etoxilo y acetilo sobre el anillo aromático así como el grupo hidroxilo en el anillo quinolínico serían los grupos funcionales responsables de contrarrestar el efecto causado por $L$. panamensis.

Aunque estos compuestos presentaron toxicidad alta, su actividad anti-Leishmania supera su citotoxicidad, razón por la cual se hace necesario continuar trabajando en la realización de modificaciones en sus estructuras para disminuir su toxicidad. El hecho de que las estirilquinolinas (1) y (2) presentaran una buena actividad leishmanicida les confiere potencial como moléculas candidatas para el desarrollo de medicamentos anti-Leishmania, y en atención a que cada vez se obtienen mejores rendimientos en su síntesis química, se convierten en una plantilla eficaz para realizar estudios posteriores de relación estructura-actividad, ensayos biodirigidos, y desarrollar moléculas activas contra el parásito con una baja citotoxicidad contra las células hospederas.

\section{CONCLUSIONES}

Mediante la reacción de condensación tipo Perkin se sintetizaron tres estirilquinolinas denominadas $(E)-2-$ etoxi-4-(2-(8-hidroxiquinolin-2-il)vinil)fenil acetato (1), (E)-4-nitro-2-(2-quinolin-2-il)vinil)fenil acetato (2), (E)-2(3-metoxiestiril)] quinolina (3), con rendimientos de $40.3 \%, 72.0 \%$ y $80.0 \%$ respectivamente. Las estirilquinolinas (1) y (2) mostraron un buen potencial leishmanicida frente al parásito de Leishmania (V) panamensis con porcentajes de inhibición de $67.5 \%$ y $74.9 \%$; estas estructuras químicas aportan bases importantes para continuar estudios tendientes a disponer de moléculas candidatas que puedan convertirse en una alternativa para el tratamiento de la leishmaniasis.

\section{AGRADECIMIENTOS}

Los autores agradecen a la Universidad de Córdoba - Colombia, por el apoyo financiero para el desarrollo de la presente investigación; al profesor Alex Sáez de la Universidad EAFIT por la toma de los espectros, y a la Universidad de Antioquía (convocatoria CIDEPRO -CIIEs). 


\section{REFERENCIAS}

Abdelwahid, m., y otros cinco autores, Synthesis, Characterization, and Antileishmanial Activity of Certain Quinoline-4carboxylic Acids, doi.org/10.1155/2019/2859637, Journal of Chemistry, 2019 (1), 1-9 (2019).

Calixto, J.T., de Morais y otros ocho autores, Phenolic composition and antiparasitic activity of plants from the Brazilian Northeast "Cerrado", dx.doi.org/10.1016/j.sjbs.2015.10.009, Saudi Journal of Biological Sciences, 23(3), 434-440 (2016).

Coinmbra, E.S., y otros siete autores, Quinoline derivatives: Synthesis, leishmanicidal activity and involvement of mitochondrial oxidative stress as mechanism of action, doi.org/10.1016/j.cbi.2016.10.017, Chemico-Biological Interactions, 260(6), 50-57 (2016).

Figueredo, F.G., y otros ocho autores, Avaliacao das potenciais atividades tripanocida e antileishmania do extrato de folhas de Piper arboreum (Piperaceae) e de suas fracoes. Rev. Cienc. Farm. Básica Apl, 35(1), 149-154 (2014).

Finney, J.D., Statistical logic in the monitoring of reactions to therapeutic drugs, Methods Inf Med, 10(4), $237-245$ (1971).

Galeano, E., O. Thomas y otros tres autores, Antiparasitic Bromotyrosine Derivatives from the Marine, Sponge Verongula rigida, doi: 10.3390/md9101902, Marine Drugs, 9(10), 1902-1913 (2011).

Instituto Nacional de Salud, Boletín Epidemiológico No 52 de la Leishmania en Colombia, (2018).

Instituto Nacional de Salud, Boletín Epidemiológico No 5 de la Leishmania en Colombia, (2019).

Insuasty, B., J. Ramirez y otros once autores, An efficient synthesis of new caffeine-based chalcones, pyrazolines and pyrazolo[3,4-b][1,4]diazepines as potential antimalarial, antitrypanosomal and antileishmanial agents, doi: org/10.1016/j.ejmech.2015.02.040, Eur J Med Chem, 93(1), 401-413 (2015).

Marin, F.J., O.L. Torres., G.G. Santafé y S.M. Robledo. Estudio fitoquímico y evaluación del potencial leishmanicida de la especie Esenbeckia litoralis (Rutaceae), doi: 10.4067/S0718-07642016000100017, Inf. Tecnol. (en linea), 27(1), 159168 (2016).

Marin, F.J., O.L. Torres., S.M. Robledo y M.E. Doria. Estudio fitoquímico y evaluación de la actividad antioxidante y leishmanicida de la especie Pilocarpus alvaradoi (Rutaceae), doi: 10.4067/S0718-076420180003001777, Inf. Tecnol. (en linea), 29(3), 177-186 (2018).

Mesa, A.M., P. Molano y otros cinco autores, Síntesis y actividades leishmanicida y citotóxica in vitro de análogos 2arilquinolinas, ISSN 0121-4004, VITAE: 15(2), 259-266 (2008).

Mrozek-Wilczkiewicz, A., E. Spaczynska y otros once autores, Design, Synthesis and In Vitro Activity of Anticancer Styrylquinolines. The p53 Independent Mechanism of Action, doi:10.1371/journal.pone.0142678, PLoS ONE (on line), 10(11), 1-14 (2015).

Oliveri, V. y G. Vecchio, 8-hidroxiquinolinas in medicinal chemistry: $A$ structural perspective, doi: 10.1016/j.ejmech.2016.05.007, European Journal of Medicinal Chemistry, 120(20), 252-274 (2016).

Pinheiro, I. M., I. Pereira y otros seis autores, Evaluation of the in vivo leishmanicidal activity of amphotericin $B$ emulgel: An alternative for the treatment of skin leishmaniasis, doi: org/10.1016/j.exppara.2016.02.010. Experimental Parasitology, 164(1), 49-55 (2016).

Pulido, S.A., D. Muñoz y otros cinco autores, Improvement of the green fluorescent protein reporter system in Leishmania spp. for the in vitro and in vivo screening of antileishmanial drugs, doi: 10.1016/j.actatropica.2011.11.015, Acta Tropica: 122(1), 36- 45 (2012).

Ribeiro, L.M., y otros ocho autores, Antileishmanial activity of a 4-hydrazinoquinoline derivative: Induction of autophagy and apoptosis-related processes and effectiveness in experimental cutaneous leishmaniasis, doi.org/10.1016/j.exppara.2018.10.007, Experimental Parasitology, 195(10), 78-86 (2018).

Santafé, G.G., E. L. Sanchez y O.L. Torres. Síntesis y actividad antimalárica de estirilquinolinas sobre Plasmodium falciparum. Información Tecnológica, 27(2), 97-104 (2016).

Sanchez, E. L., G.G. Santafé y otros tres autores, Compuestos sintéticos del tipo de estirilquinolinas con actividad leishmanicida y citotóxica. Biomédica, 34(4), 605-611 (2014).

Soto, A.F., J.P. Meneses., J. Saez y M. Camargo, Evaluación de citotoxicidad de nuevos análogos de estirilquinolinas en células leucemoides Jurkat, Actual Biol, 33(95), 165-172 (2011).

Torres, O.L., R.D. Espinosa., A.A. Saez y G.G. Santafé, Evaluación de la actividad antimalárica y citotóxica de estirilquinolinas obtenidas a partir de 8-hidroxiquinaldina con aldehídos aromáticos, doi: 10.4067/S071807642014000600012, Inf. Tecnol, 25(6), 91- 100 (2014).

World Health Organization (WHO), Leishmaniasis. Epidemiological report of the Americas. º 6, February (2018).

World Health Organization (WHO), Control of the leishmaniases: report of a meeting of the WHO Expert Commitee on the Control of Leishmaniases. Technical report 949 (2010). 
